\title{
Sympathetic crashing acute pulmonary oedema (SCAPE), dealing with the worst heart failure
}

\author{
Ranasinghe AM ${ }^{1}$, Lakruwan GLA ${ }^{1}$, Kumara KKJ ${ }^{2}$ \\ ${ }^{I}$ National Hospital, Colombo, Sri Lanka. \\ ${ }^{2}$ Emergency Treatment Centre, Teaching Hospital Karapitiya, Sri Lanka. \\ Correspondence: Dr. Achala Ranasinghe \\ e-mail: achalarsnet@gmail.com \\ https://orcid.org/0000-0002-9847-5287
}

\section{Introduction}

Sympathetic crashing acute pulmonary oedema (SCAPE) is the most severe form of the spectrum of acute heart failure syndrome (AHFS), characterised by excessive sympathetic activation which results in very high blood pressure and redistribution of extracellular fluid causing severe pulmonary oedema, respiratory failure and death (1). This particular subset of patients present with acute onset and rapid progression of symptoms soon develops into a life-threatening condition within minutes to hours. Diagnosis of SCAPE is purely clinical and if effectively managed, the outcome is usually $\operatorname{good}(2)$.

This case report documents an acutely deteriorating patient with SCAPE preceding unstable angina who was successfully treated with high dose nitroglycerin and non-invasive positive pressure ventilation (NIPPV) obviating the need for tracheal intubation and intensive care unit (ICU) admission.

\section{Case presentation}

We present a 66-year-old male, with history of unstable angina, who presented to the emergency treatment unit (ETU), Teaching Hospital Karapitiya with shortness of breath, which progressed rapidly over 2 hours. It was associated with chest tightness, sweating and a few episodes of vomiting. His last recorded blood pressure at the clinic was 130/80 mmHg.

On admission to the ETU, he was restless, agitated and profusely diaphoretic. He was severely dyspnoeic with a respiratory rate of $44 / \mathrm{min}$ and he had a feeling of suffocation. Auscultation revealed bilateral coarse crepitations up to the level of clavicles and his oxygen saturation $\left(\mathrm{SPO}_{2}\right)$ was $82 \%$ with high flow oxygen. He had a pulse rate of 130 beats per minute and his blood pressure recorded was $230 / 110 \mathrm{mmHg}$.

Arterial blood gas showed $\mathrm{pH}-7.2, \mathrm{PaO}_{2}-45$ mmHg (with $\mathrm{FiO}_{2}-0.8$ - 0.9), $\mathrm{PCO}_{2}-46 \mathrm{mmHg}$, $\mathrm{HCO}_{3}-18 \mathrm{mmol} / \mathrm{L}$, serum lactate $-6 \mathrm{mmol} / \mathrm{L}$, indicating a mixed acidosis with type 2 respiratory failure. Bedside ultrasound scan further suggested the diagnosis of pulmonary oedema (presence of $>\mathrm{B}$ lines on one intercostal space in bilateral lung fields $-B$ profile).

Intravenous nitroglycerin and NIPPV were commenced immediately. A loading dose of intravenous nitroglycerin $400 \mathrm{mics} / \mathrm{min}$ was given over two minutes and followed by $100 \mathrm{mics} / \mathrm{min}$ infusion. Manual blood pressure was checked in every 2 minutes to adjust the rate of infusion. After 10 minutes of treatment, a blood pressure of $150 / 80 \mathrm{mmHg}$ was reached and the infusion was slowly tailed off. Oral (NG) captopril $25 \mathrm{mg}$ was given. Patient's respiratory distress, oxygen saturation, restlessness and conscious level were dramatically improved within 10 minutes of treatment. Later he was treated for unstable angina and admitted to the acute side of a medical ward. 


\section{Discussion}

In SCAPE, as opposed to other AHF syndromes, the main aim is to achieve a rapid afterload reduction to block the vicious cycle resulted from the sympathetic surge, and thereby, to improve cardiac output and diastolic dysfunction. Therefore, initiation of nitroglycerin with a high dose (with vasodilator effect) is advised as opposed to gradual up-titration (3). Commonly practiced protocol is a loading dose of $200-500 \mathrm{mics} / \mathrm{min}$ for 2 minutes followed by an infusion of $100 \mathrm{mics} / \mathrm{min}$ with rapid titration till systolic blood pressure reaches 140 $\mathrm{mmHg}$ (or previous normal value) and clinical improvement (4).

We did not start loop diuretics during early management in the ETU. There were principally two reasons for it. In this category of patients, due to increased afterload, the renal perfusion is compromised and therefore, the amount of drug reaching the kidneys is decreased resulting in late diuretic action (5). Further, its venodilatory effect is not proven in the literature among this group of patients. Therefore, it was withheld till improvement in afterload and renal perfusion was achieved by other medications. Secondly, frusemide administration within 90 minutes of onset has shown to increase the mortality in acute decompensated heart failure (5). It is thought to be due to initial activation of renin-angiotensin-aldosterone system (RAAS) which causes an increase in afterload and worsening of the primary pathology (5).

\section{Conclusions and recommendations}

In SCAPE, early and effective treatment is proven to reduce the incidence of tracheal intubation, ICU admission and mortality. High dose nitroglycerin and NIPPV remains the mainstay of early management. Early administration of diuretics, although commonly used, is not supported by good scientific evidence.

\section{References}

1. Rimoldi SF, Yuzefpolskaya M, Allemann Y, Messerli F. Flash pulmonary oedema. Prog Cardiovasc Dis, 2009 Nov-Dec; 52(3): 249-59.

2. Naman Agrawal, Akshay Kumar, Praveen Aggarwal, Nayer Jamshed. Sympathetic crashing acute pulmonary oedema. Indian J Crit Care Med, 2016 Dec; 20(12): 71923.

3. Levy P, Compton S, Welch R, Delgado G, Jennett A. Treatment of severe decompensated heart failure with high-dose intravenous nitroglycerin: a feasibility and outcome analysis. Ann Emerg Med, 2007 Aug; 50(2): 144-52.

4. Weingart S. Emcrit Podcast 1, Sympathetic Crashing Acute Pulmonary Edema.

5. Felker GM, O'Connor CM, Braunwald E. Loop diuretics in acute decompensated heart failure: necessary? Evil? A necessary evil?. Circ Heart Fail, 2009 Jan; 2(1): 56-62. 